\title{
Ethanol-activated CaMKII signaling induces neuronal apoptosis through Drp1-mediated excessive mitochondrial fission and JNK1-dependent NLRP3 inflammasome activation.
}

\section{CURRENT STATUS: UNDER REVIEW}

(8) Cell Communication \& Signaling $\triangle B M C$

Jae Ryong Lim

Seoul National University College of Veterinary Medicine

Hyun Jik Lee

Seoul National University College of Veterinary Medicine

Young Hyun Jung

Seoul National University College of Veterinary Medicine

Jun Sung Kim

Seoul National University College of Veterinary Medicine

Chang Woo Chae

Seoul National University College of Veterinary Medicine

Seo Yihl Kim

Seoul National University College of Veterinary Medicine

Ho Jae Han

Seoul National University College of Veterinary Medicine

- hjhan@snu.ac.krCorresponding Author

ORCiD: https://orcid.org/0000-0002-0657-1766

DOI:

10.21203/rs.2.18316/v1

\section{SUBJECT AREAS}

Cell Communication and Signaling

\section{KEYWORDS}

Ethanol, NMDA receptor, NLRP3 inflammasome, CaMKII, JNK1, Drp1, Caspase-1, Mitophagy, Neuronal apoptosis 
Abstract

Background: Neurodegeneration is a representative phenotype of patients with chronic alcoholism. Ethanol-induced calcium overload causes NLRP3 inflammasome formation and an imbalance in mitochondrial dynamics, closely associated with the pathogenesis of neurodegeneration. However, how calcium regulates this process in neuronal cells is poorly understood. Therefore, the present study investigated the detailed mechanism of calcium-regulated mitochondrial dynamics and NLRP3 inflammasome formation in neuronal cells by ethanol.

Methods: In this study, we used the SK-N-MC human neuroblastoma cell line. To confirm the expression level of the mRNA and protein, real time quantitative PCR and western blot were performed. Co-immunoprecipitation and Immunofluorescence staining were conducted to confirm the complex formation or interaction of the proteins. Flow cytometry was used to analyze intracellular calcium, mitochondrial dysfunction and neuronal apoptosis.

Results: Ethanol increased cleaved caspase-3 levels and mitochondrial reactive oxygen species (ROS) generation associated with neuronal apoptosis. In addition, ethanol increased PKA activation and CREB phosphorylation, which increased NMDA receptor (NMDAR) expression. Ethanol-increased NMDAR induced intracellular calcium overload and CaMKII activation leading to phosphorylation of dynamin-related protein 1 (Drp1) and JNK1. Drp1 phosphorylation promoted Drp1 translocation to the mitochondria, resulting in excessive mitochondrial fission, mitochondrial ROS accumulation, and loss of mitochondrial membrane potential, which was recovered by Drp1 inhibitor pretreatment. Ethanolinduced JNK1 phosphorylation activated the NLRP3 inflammasome that induced caspase-1 dependent mitophagy inhibition, thereby exacerbating ROS accumulation and causing cell death. Suppressing caspase-1 induced mitophagy and reversed the ethanol-induced apoptosis in neuronal cells.

Conclusions: Our results demonstrated that ethanol upregulated NMDAR-dependent CaMKII phosphorylation which is essential for Drp1-mediated excessive mitochondrial fission and the JNK1induced NLRP3 inflammasome activation resulting in neuronal apoptosis.

Full Text

Due to technical limitations, full-text HTML conversion of this manuscript could not be completed. 
However, the manuscript can be downloaded and accessed as a PDF.

Table

Due to technical limitations, Table 1 is only available as a download in the supplemental files section Figures 
A

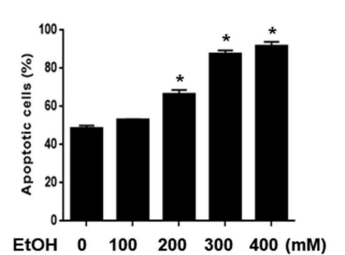

B

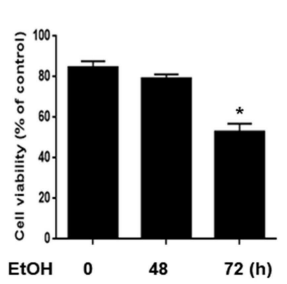

C
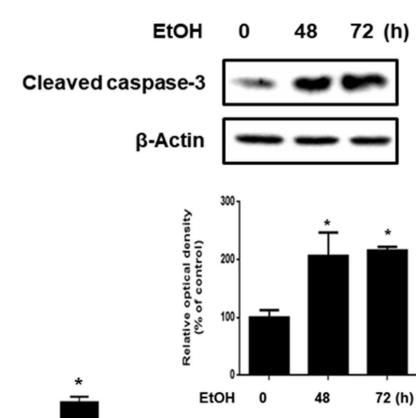

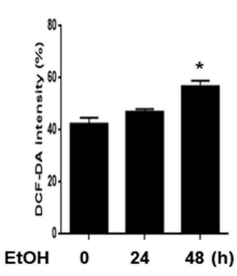

F

E
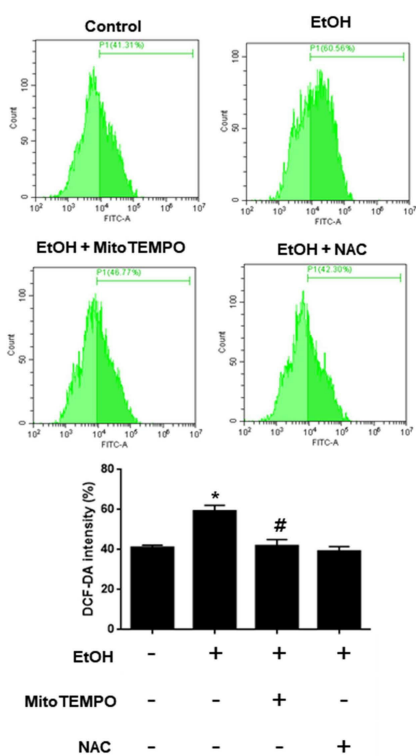

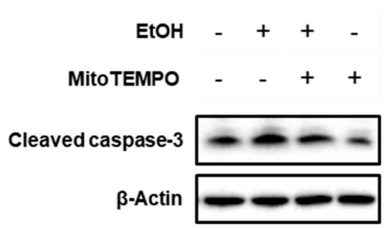

$\beta$-Actin

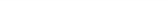

MitoTEMPO

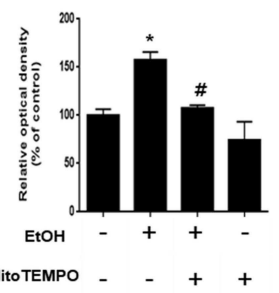

G
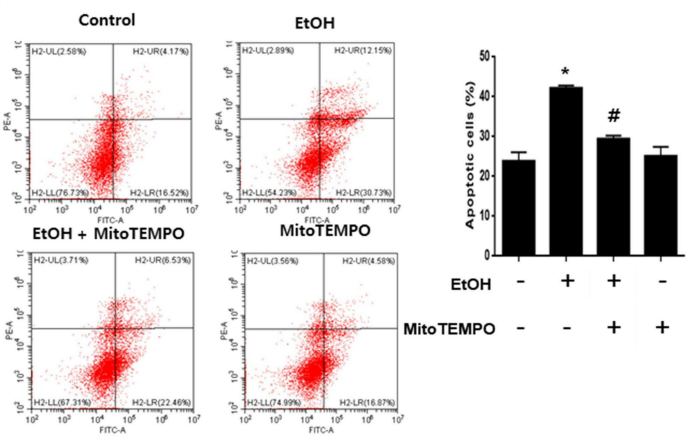
Figure 1

Role of ethanol-induced mitochondrial ROS accumulation in neuronal cell death. A SK-N-MC cells were incubated with various concentration of EtOH (100-400 mM) for $72 \mathrm{~h}$.

Quantitative analysis of the fold changes of apoptotic cells were measured by using annexin V/PI staining with flow cytometry. Data are presented as a mean \pm S.E.M. $n=4$. B Cells were exposed to EtOH (200 mM) for 0-72 h. Cell viability was measured by trypan blue exclusion assay. Data are presented as a mean \pm S.E.M. $n=4$. C Cells were exposed to EtOH (200 mM) for 0-72 h. Cleaved caspase-3 was detected by western blot. Data are presented as a mean \pm S.E.M. $n=3$. D Cells were treated with EtOH $(200 \mathrm{mM})$ in a timedependent manner, ROS measurement by H2DCF-DA was conducted by flow cytometry. Data are presented as a mean \pm S.E.M. $n=3$. E Cells were pretreated with mitoTEMPO ( 2 $\mu \mathrm{M})$ and NAC (5 mM) for 30 min and incubated with ETOH (200 mM) for $48 \mathrm{~h}$. Then H2DCFDA was incubated for 30 min to detect ROS and it was measured by using flow cytometry. Data are presented as a mean \pm S.E.M. $n=3$. F Cells were pretreated with mitoTEMPO $(2 \mu \mathrm{M})$ for $30 \mathrm{~min}$, and then exposed to EtOH (200 mM) for $48 \mathrm{~h}$. Western blotting was conducted to determine the levels of cleaved caspase-3. Data are presented as a mean \pm S.E.M. $n=4$. G Cells were pretreated with mitoTEMPO $(2 \mu M)$ for 30 min prior to EtOH treatment for $72 \mathrm{~h}$. Apoptotic cells were detected by annexin V/ PI staining. Data are presented as a mean \pm S.E.M. $n=3$. All blot images are representative. ${ }^{*} p<0.05$ versus control, $\# p<0.05$ versus EtOH. 
A

B

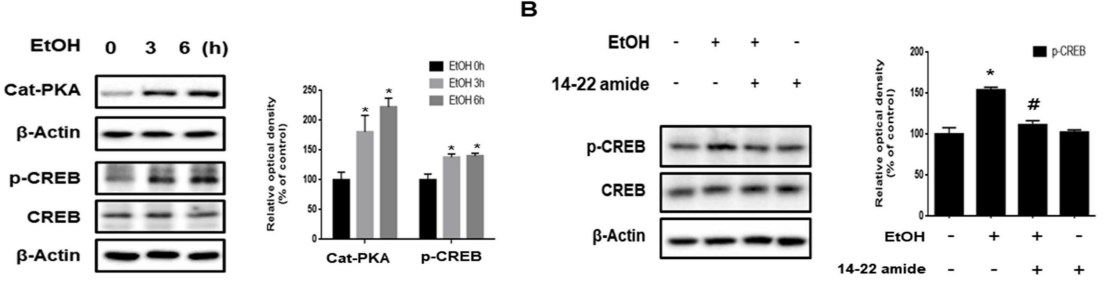

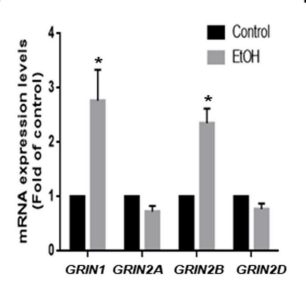

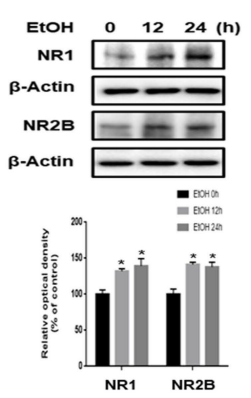

E
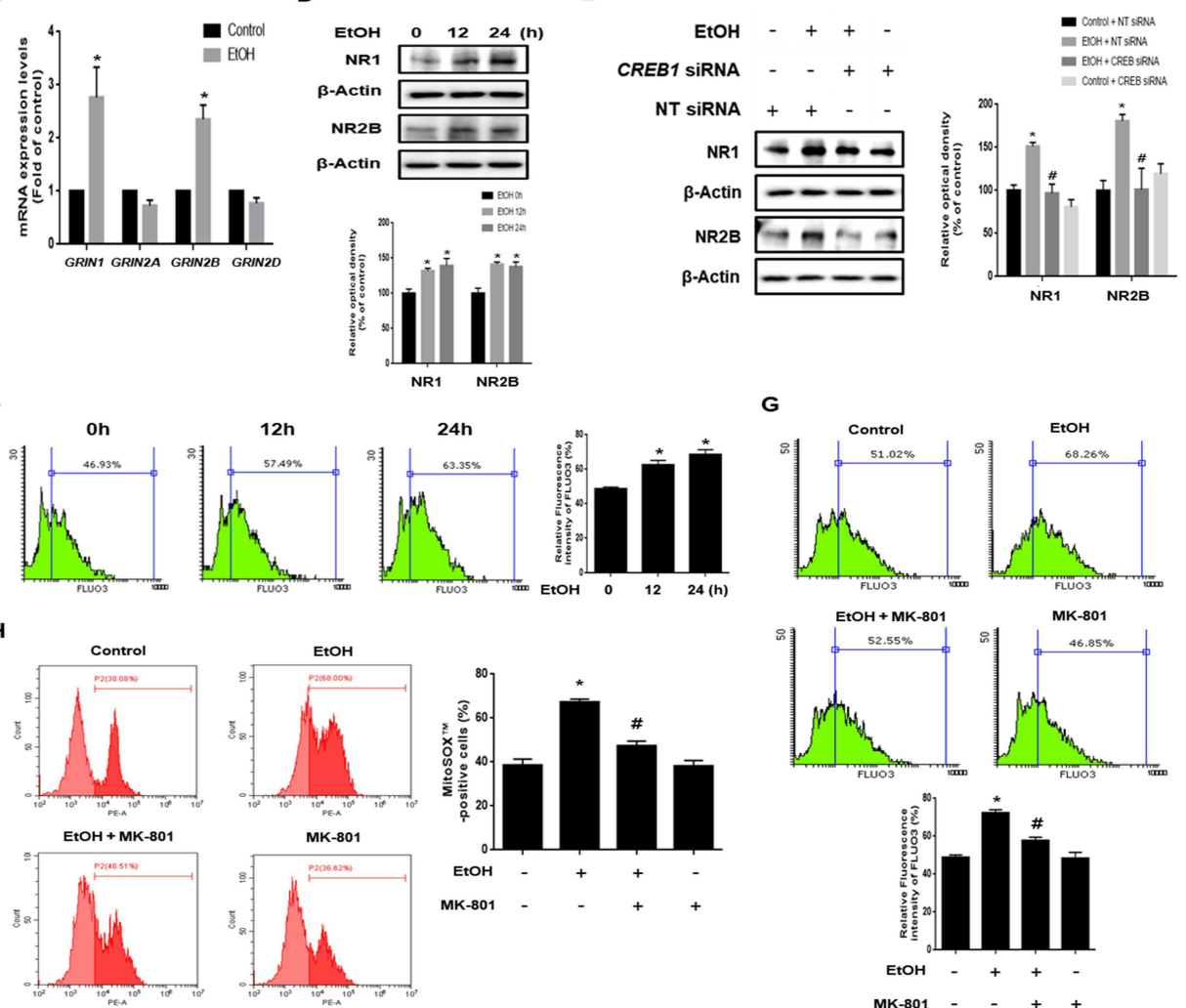

I
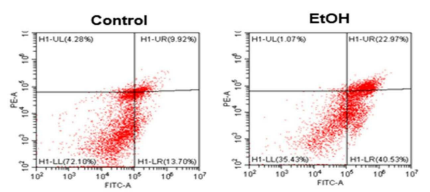

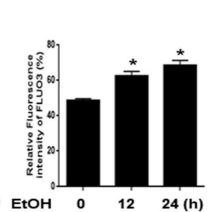

G
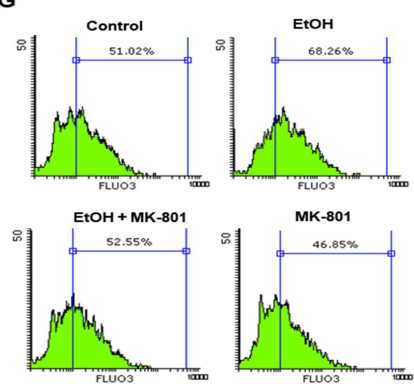

мк-80
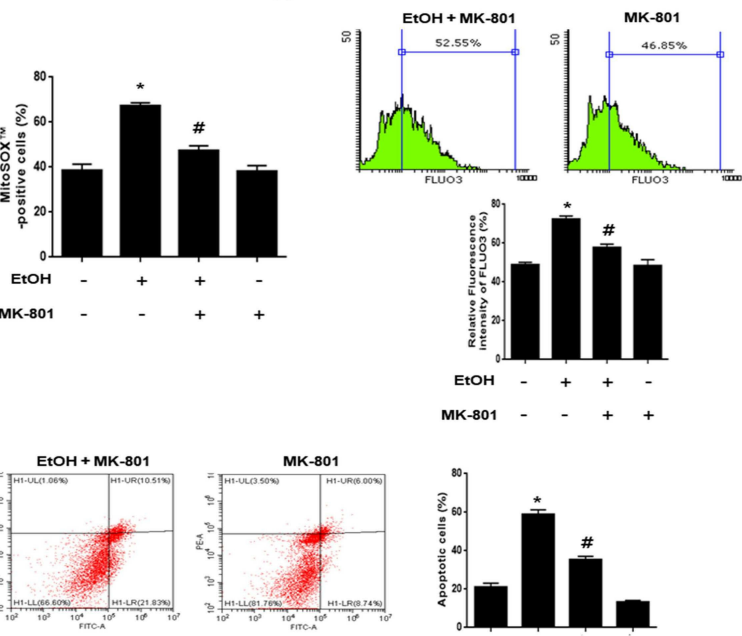

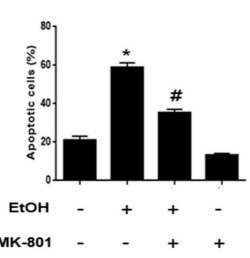

Figure 2 
Role of PKA/CREB pathway in ethanol-induced NMDAR expression and intracellular calcium overload. A SK-N-MC cells were treated with EtOH $(200 \mathrm{mM})$ for various time $(0-6 \mathrm{~h})$. CatPKA, p-CREB (Ser 113) and CREB were detected by western blot. $\beta$-Actin was used as a loading control. $n=3$. B Cells were pretreated with $14-22$ amide ( $1 \mu \mathrm{M})$ for 30 min before EtOH treatment. CREB and p-CREB (Ser 113) were analyzed by western blotting. $\beta$-Actin was used as a loading control. $\mathrm{n}=4$. C Cells were treated with EtOH (200 mM) for $12 \mathrm{~h}$. mRNA expressions of GRIN1, GRIN2A, GRIN2B, and GRIN2D were analyzed by quantitative real time PCR. Data were normalized by the ACTB mRNA expression level. $n=4$. D Cells were exposed to EtOH (200 mM) for 0-24 h. NR1 and NR2B were detected by western blot. $\mathrm{n}=4$. E Cells were transfected with CREB siRNA or NT siRNA for $24 \mathrm{~h}$ prior to ethanol exposure for $12 \mathrm{~h} . \mathrm{NR} 1$ and NR2B expressions were measured by western blotting. $\mathrm{n}=3 .{ }^{*} \mathrm{p}<0.05$ versus control with NT siRNA transfection, $\# p<0.05$ versus EtOH with NT siRNA transfection F Cells were loaded with Fluo 3-AM $(2 \mu \mathrm{M})$ for $30 \mathrm{~min}$ and then exposed to EtOH for 0-24 h. The amount of intracellular calcium was measured by using flow cytometry. $\mathrm{n}=$ 3. G Cells were loaded with Fluo 3-AM $(2 \mu \mathrm{M})$ for $30 \mathrm{~min}$, subsequently pretreated with MK$801(10 \mu \mathrm{M})$ for 30 min prior to EtOH treatment. $\mathrm{n}=4$. H The population of MitoSOX ${ }^{\mathrm{TM}}$ positive cells was measured by flow cytometry. $n=3$. I Apoptotic cells were measured by annexin V/PI analysis assay. All data are presented as a mean \pm S.E.M. $n=3$. All blot images are representative. ${ }^{*} p<0.05$ versus control, $\# p<0.05$ versus EtOH. 
A

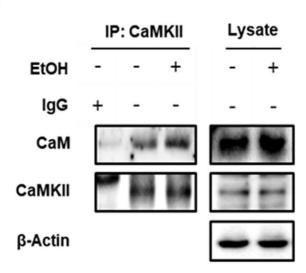

C
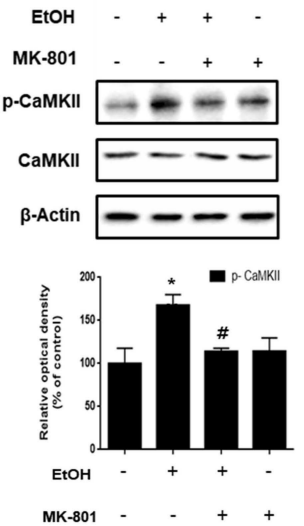

F
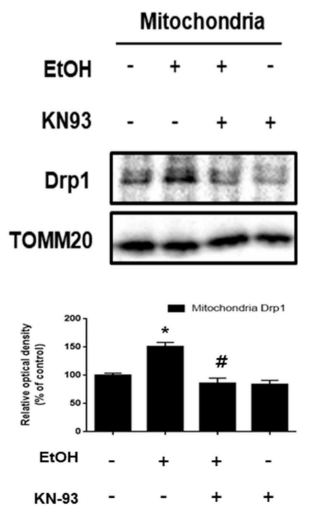

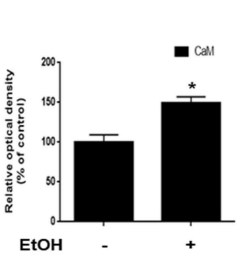

D
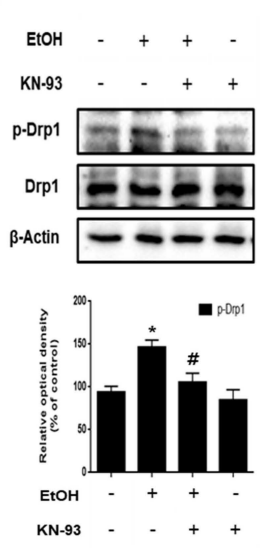

G

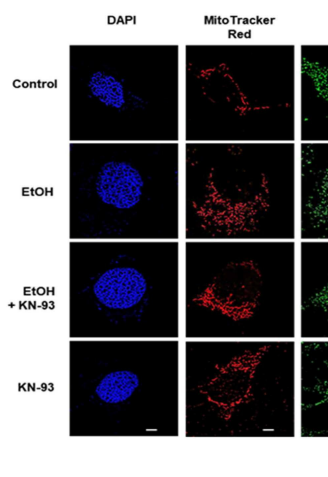

E
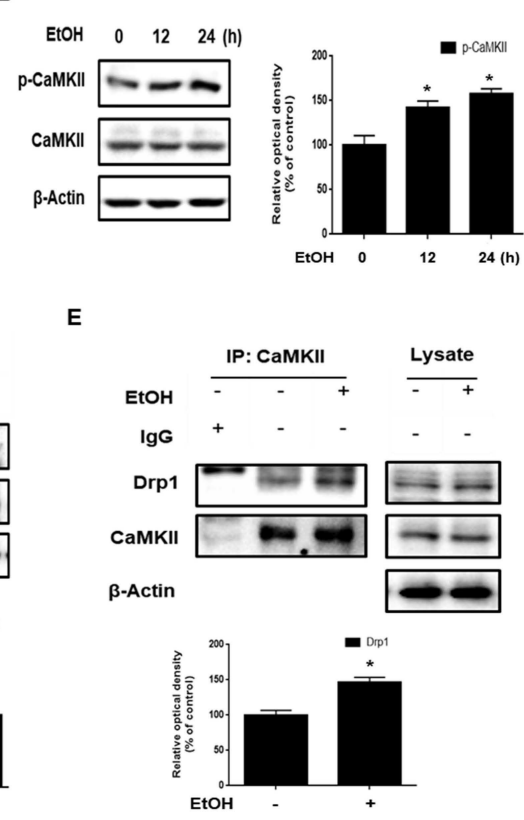

$1-2$

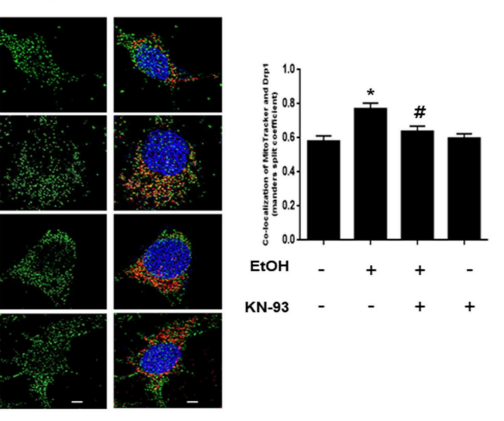

Figure 3 
Ethanol-induced CaMKII activation promotes translocation of Drp1 to the mitochondria. A

SK-N-MC cells were incubated EtOH $(200 \mathrm{mM})$ for $12 \mathrm{~h}$ and then harvested. CaMKII was immunoprecipitated with an anti-CaM, anti-CaMKII antibodies (left). The expression of CaM, CaMKII, and $\beta$-Actin in total cell lysates is shown (right). $n=3$. B Cells were treated with EtOH for various times (0-24 h). CaMKII and p-CaMKII (Thr 286) were analyzed by western blot. $\beta$-Actin was used as a loading control. $n=4$. C Cells were pretreated with MK-801 (10 $\mu \mathrm{M})$ for 30 min prior to EtOH treatment for $24 \mathrm{~h}$. p-CaMKII (Thr 286) and CaMKII were detected by western blot. $n=4$. D Cells were pretreated with $K N-93(1 \mu M)$ for 30 min prior to EtOH treatment for $24 \mathrm{~h}$. Drpl and p-Drpl (Ser616) were detected by western blot. $\beta$ Actin was used as a loading control. $n=4$. E Cells were incubated EtOH $(200 \mathrm{mM})$ for $24 \mathrm{~h}$ and then harvested. CaMKII was immunoprecipitated with anti- CaMKII and anti-Drp1 antibodies (left). The expression of CaMKII, Drp1 and $\beta$-Actin in total cell lysates is shown (right). $\mathrm{n}=3$. F Cells were pretreated with $\mathrm{KN}-93(1 \mu \mathrm{M})$ for $30 \mathrm{~min}$ prior to EtOH treatment for $48 \mathrm{~h}$. Mitochondrial and cytosolic fractions were mentioned in methods. Drp1 was detected by western blot. Anti- TOMM20 was used as mitochondria marker. $n=3$. G Cells were pre-treated with $\mathrm{KN}-93(1 \mu \mathrm{M})$ for 30 min prior to $\mathrm{EtOH}$ treatment for $24 \mathrm{~h}$ and immunostained with Drpl antibody and Mitotracker ${ }^{\mathrm{TM}}$. Co-localization of Drp1 (green) and Mitotracker $^{\mathrm{TM}}$ (red) was visualized with SRRF imaging system. Scale bars are $8 \mu \mathrm{m}$ (magnification, $\times 1000) . n=4$. All data are presented as a mean \pm S.E.M. All blot and immunofluorescence images shown are representative. ${ }^{*} p<0.05$ versus control, $\# p<0.05$ versus EtOH. 
A

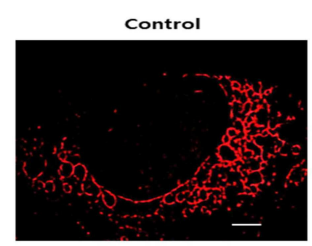

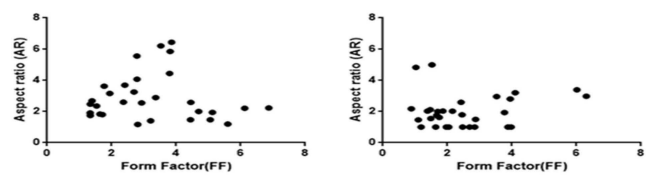

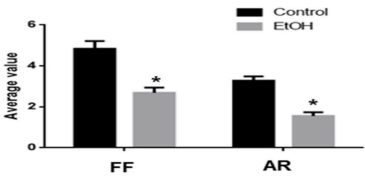

C
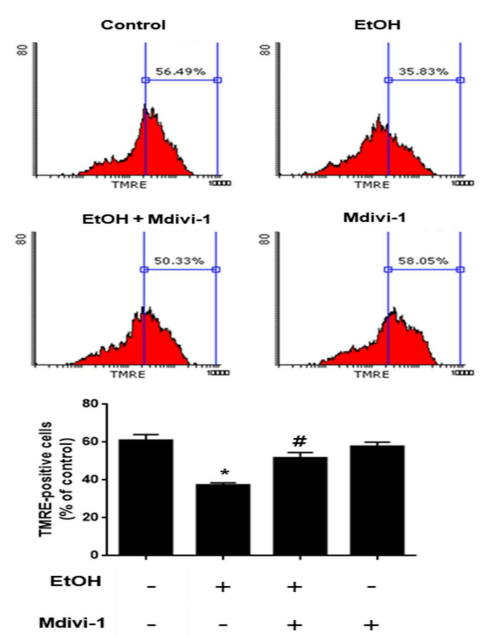
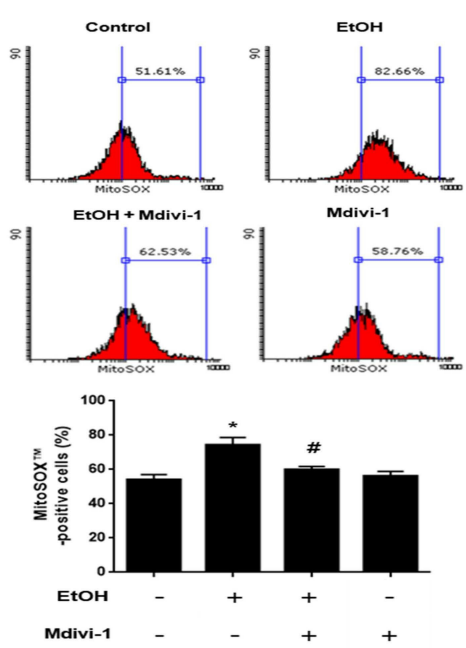

D
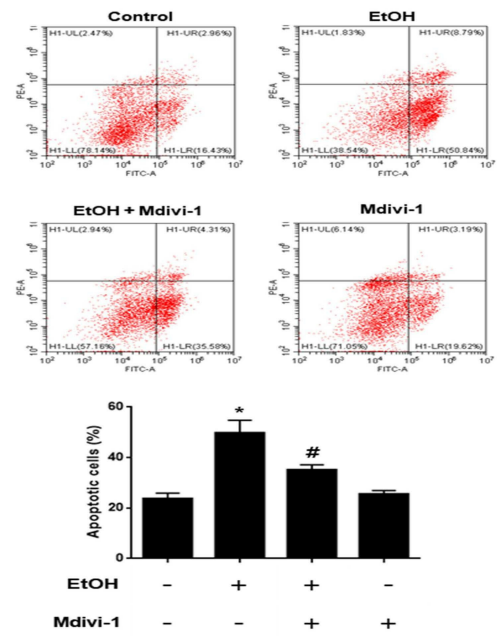

Figure 4 
Ethanol-induced excessive mitochondrial fission leads to mitochondrial dysfunction. A SK-NMC cells were exposed to EtOH for $48 \mathrm{~h}$ and then loaded with Mitotracker ${ }^{\mathrm{TM}}$ Red (200 $\mathrm{nM}$ ). Representative images were visualized with SRRF imaging system. Scattered plots and average values for form factor (FF) and aspect ratio (AR) were shown on below. Data are presented as a mean \pm S.E.M. $n=10$. Scale bars are $8 \mu$ m (magnification, $\times 1000$ ). B Cells were pretreated with Mdivi-1 (1 $\mu \mathrm{M})$ for 30 min before EtOH treatment for $48 \mathrm{~h}$. Cells were stained with MitoSOX ${ }^{\mathrm{TM}}$. The population of MitoSOX ${ }^{\mathrm{TM}}$-positive cells was measured by flow cytometry. Data are presented as a mean \pm S.E.M. $n=3$. C Cells were stained with tetramethylrhodamine ethyl ester (TMRE). The population of TMRE-positive cells was measured by flow cytometry. Data are presented as a mean \pm S.E.M. $n=3$. D Cells were pretreated with Mdivi-1 ( $1 \mu \mathrm{M})$ for 30 min before EtOH treatment for $72 \mathrm{~h}$. Apoptotic cells were measured by annexin V/PI analysis assay. Data are presented as a mean \pm S.E.M. $n=$ 3. All blot and immunofluorescence images shown are representative. $* p<0.05$ versus control, $\# p<0.05$ versus EtOH. 


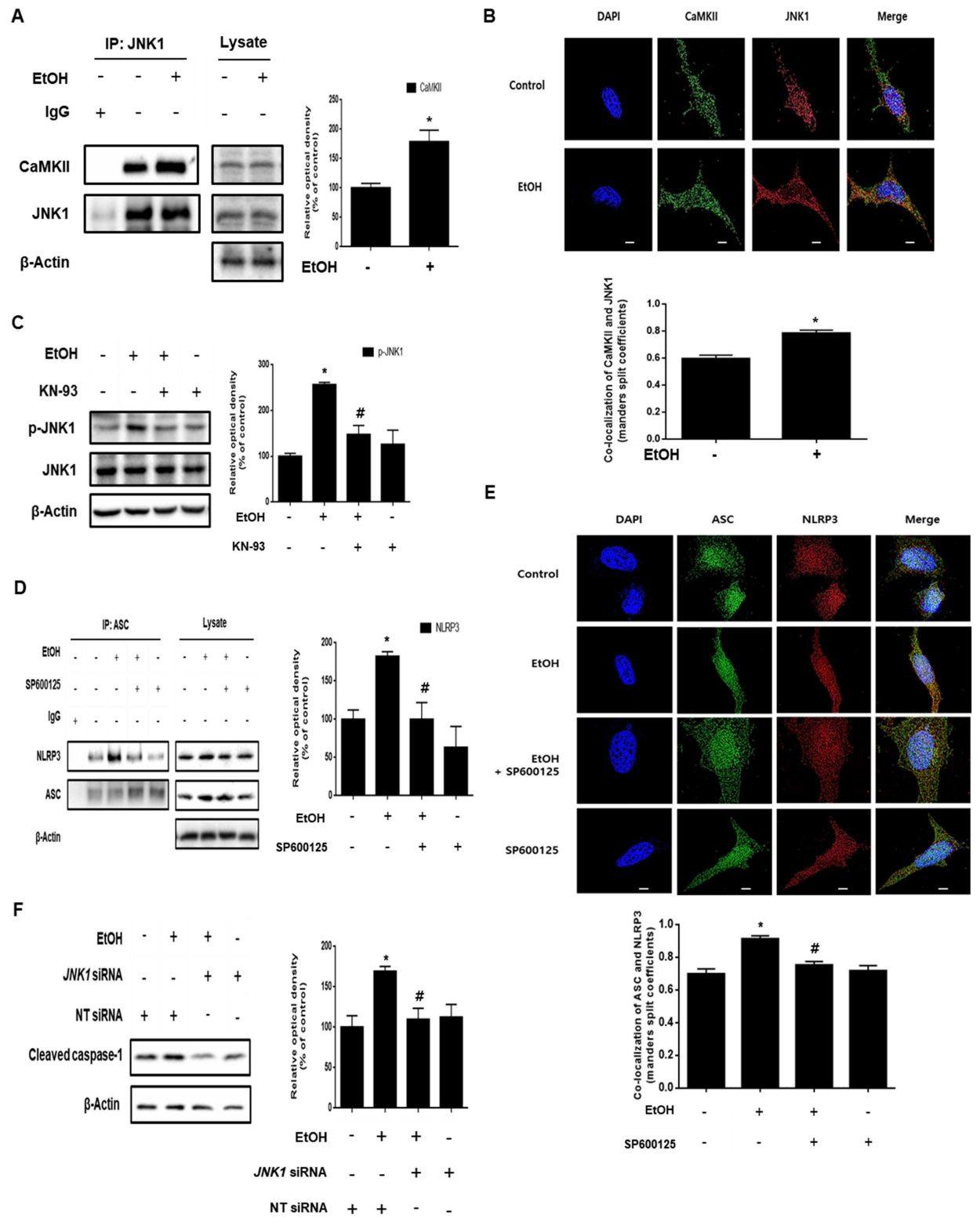

Figure 5 
Role of CaMKII/JNK1 pathway in ethanol-induced inflammasome activation. A SK-N-MC cells were incubated EtOH (200 mM) for $24 \mathrm{~h}$ and then harvested. JNK1 was immunoprecipitated with anti-JNK1 and anti- CaMKII antibodies (left). The expression of JNK1, CaMKII, and $\beta$-Actin in total cell lysates is shown (right). Data are presented as a mean \pm S.E.M. $n=3$. B Cells were incubated with EtOH for $24 \mathrm{~h}$ and immunostained with CaMKII and JNK1 antibodies. Colocalization of CaMKII (green) and JNK1 (red) was visualized with SRRF imaging system. Scale bars are $8 \mu \mathrm{m}$ (magnification, $\times 1000) . \mathrm{n}=5$. C Cells were pre-treated with KN-93 (1 $\mu \mathrm{M}$ ) for 30min prior to EtOH treatment for $24 \mathrm{~h}$. JNK1 and phosphorylated JNK1 (Thr 183/ Tyr 185) were analyzed by western blotting. $\beta$-Actin was used as a loading control. Data are presented as a mean \pm S.E.M. $n=4$. D Cells were pre-treated with SP600125 (10 $\mu$ M) for 30 min prior to EtOH treatment for 48 h. ASC was immunoprecipitated with anti-ASC and antiNLRP3 antibodies (left). The expression of ASC, NLRP3 and $\beta$ - Actin in total cell lysates is shown (right). Data are presented as a mean \pm S.E.M. $n=3$. E Cells were immunostained with ASC and NLRP3 antibodies. Co-localization of ASC (green) and NLRP3 (red) was visualized with SRRF imaging system. Scale bars are $8 \mu \mathrm{m}$ (magnification, $\times 1000$ ). $\mathrm{n}=4$. F Cells were transfected with JNK1 siRNA or NT siRNA for 24 h prior to ethanol exposure for 48 h. Cleaved caspase- 1 expression was measured by western blotting. $\beta$-Actin was used as a loading control. Data are presented as a mean \pm S.E.M. $n=3$. All blot and immunofluorescence images shown are representative. ${ }^{*} p<0.05$ versus control, $\# p<0.05$ versus EtOH. 
A

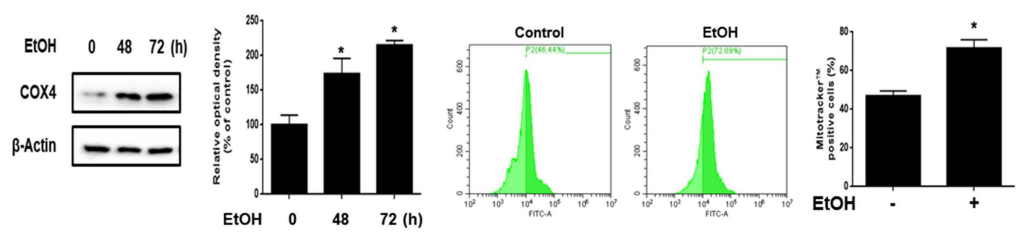

B

C

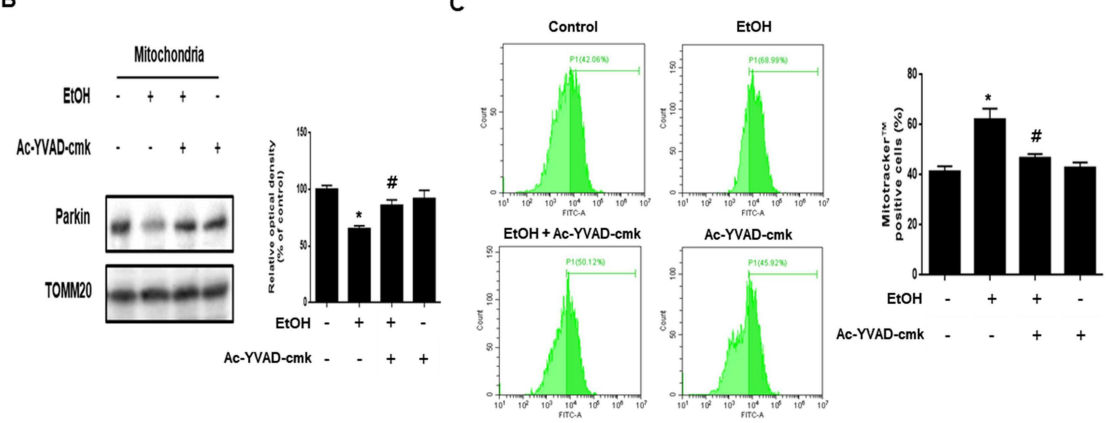

D

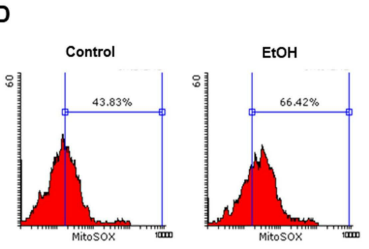

E
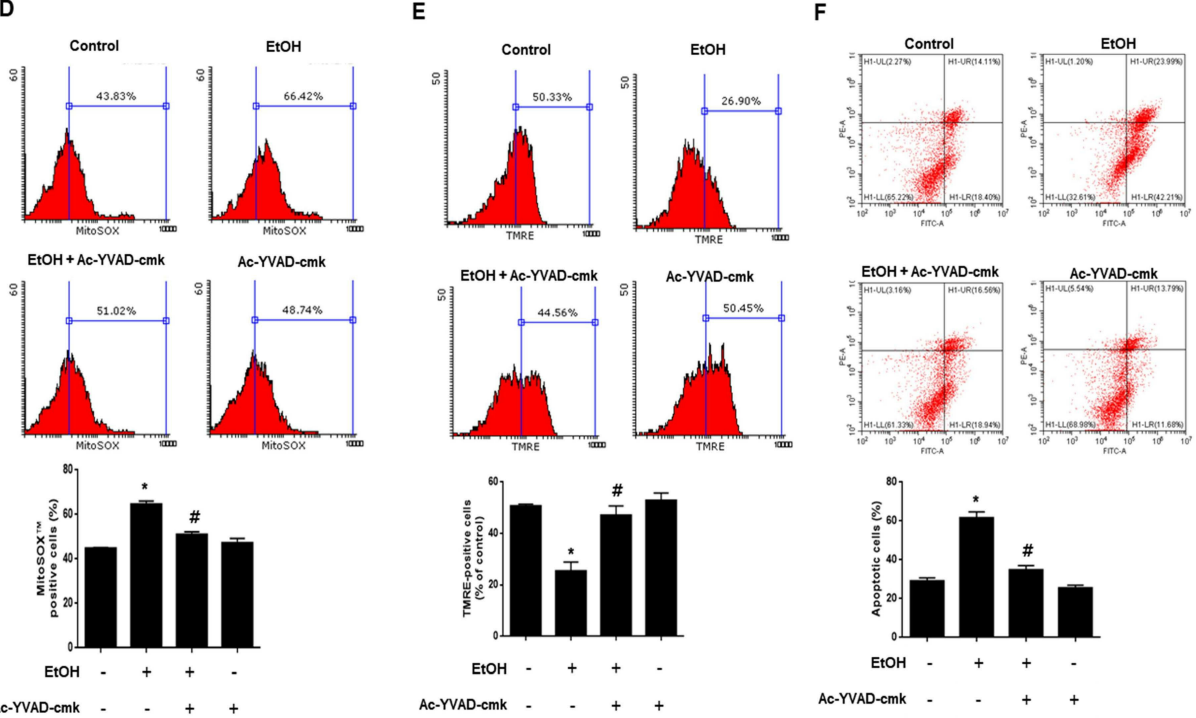

Figure 6 
Role of ethanol-induced caspase-1 activation in parkin-mediated mitophagy inhibition. A Cells were exposed to EtOH (200 mM) for 0-72 h. COX4 was detected by western blot. $\beta$ Actin was used as a loading control. The population of Mitotracker ${ }^{\mathrm{TM}}$-positive cells was measured by flow cytometry. Data are presented as a mean \pm S.E.M. $n=3$. B parkin and TOMM20 with mitochondrial fractionized samples were detected by western blot. Data are presented as a mean \pm S.E.M. $n=4$. C Cells were pre-treated with AC-YVAD-cmk (10 $\mu M)$ for 30 min prior to EtOH treatment for 48 h. Cells were stained with Mitotracker ${ }^{\mathrm{TM}}$. The population of Mitotracker ${ }^{T M}$-positive cells was measured by flow cytometry. Data are presented as a mean \pm S.E.M. $n=4$. D The population of MitoSOX ${ }^{\mathrm{TM}}$-positive cells was measured by flow cytometry. Data are presented as a mean \pm S.E.M. $n=3$. E The population of TMRE-positive cells was measured by flow cytometry. Data are presented as a mean \pm S.E.M. $n=4 . F$ Cells were pretreated with Ac-YVAD-cmk $(10 \mu M)$ for 30 min before ethanol treatment for $72 \mathrm{~h}$. Apoptotic cells were measured by annexin V/PI analysis assay. Data are presented as a mean \pm S.E.M. $n=3$. All blot images are representative. $* p<0.05$ versus control, $\# p<0.05$ versus EtOH.

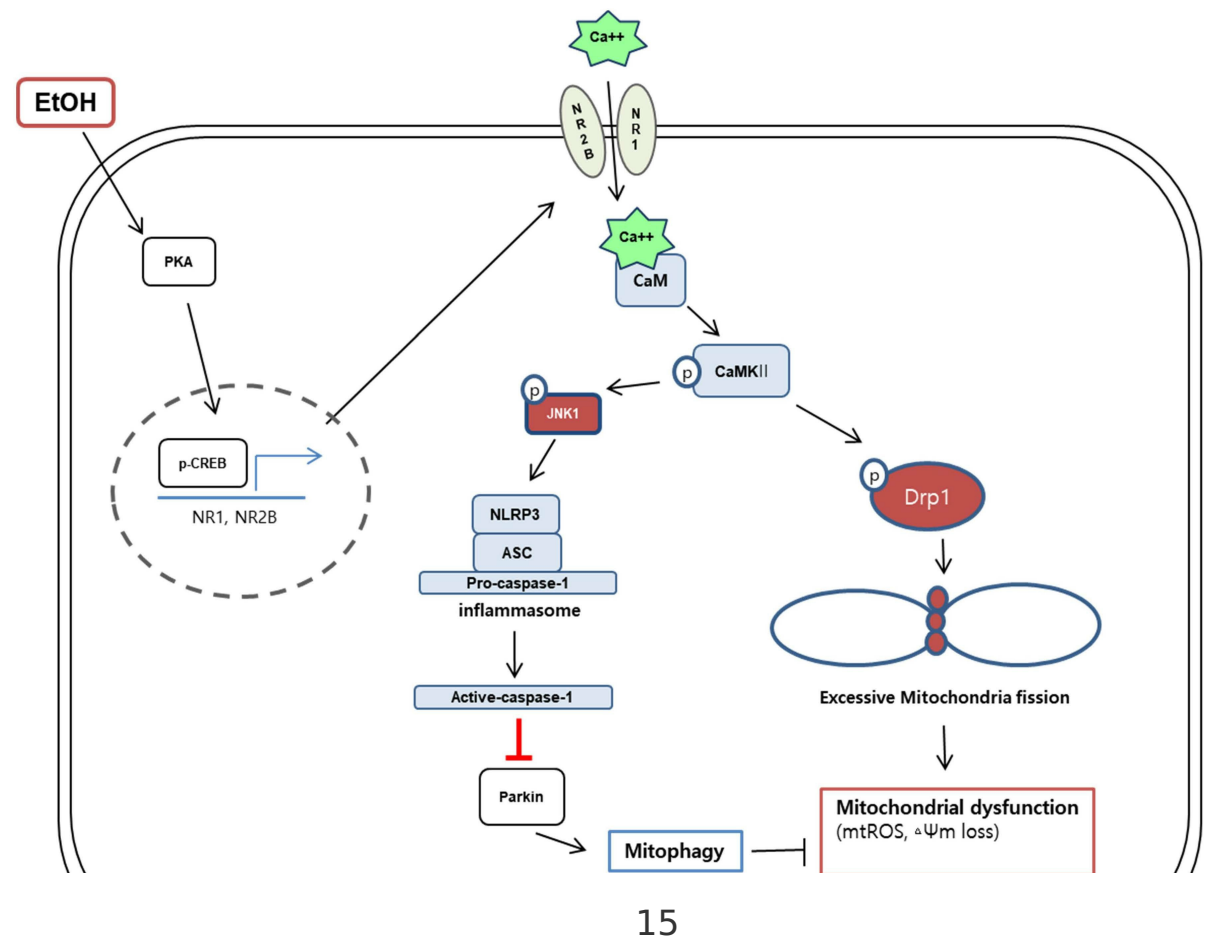




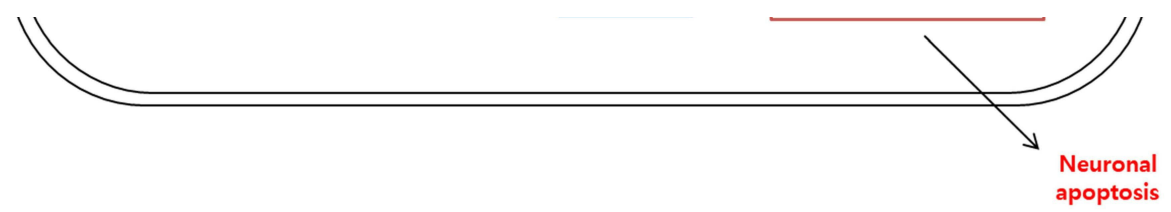

Figure 7

The schematic model for mechanism involved in EtOH-induced neuronal apoptosis. EtOHinduced NLRP3 inflammasome activation inhibits the mitophagy, which increases the accumulation of mitochondrial ROS by excessive fission leading to neuronal apoptosis.

\section{Supplementary Files}

This is a list of supplementary files associated with this preprint. Click to download. 20191129 Table.pdf

20191129 Additional file.pdf 